\title{
Multi-Sensor Fall Detection for Smartphones
}

\author{
Noemi Biancone, Chiara Bicchielli*, Fernando Ferri and Patrizia Grifoni \\ IRPPS-CNR, Via Palestro 32, Italy
}

*Corresponding author: Chiara Bicchielli, IRPPS-CNR, Via Palestro 32, Rome, Italy

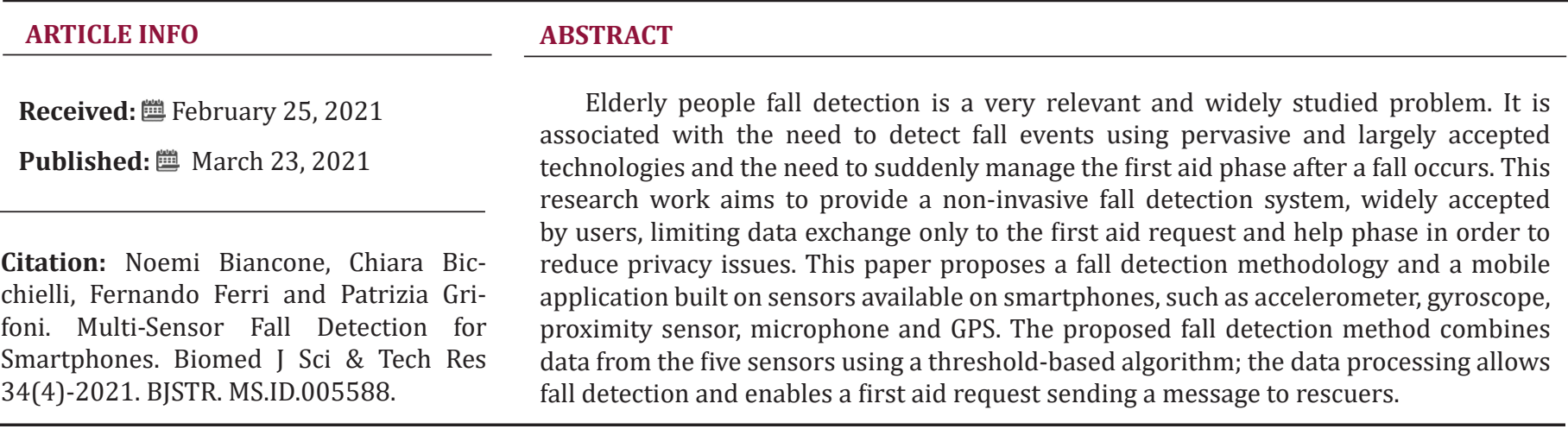

\section{Introduction}

Among unintentional injuries, the World Health Organization [1] identified falls as part of the leading causes of death and, in less dangerous cases, provoking immobility and premature nursing home placement. About 37.3 million falls occur every year that are serious enough to require medical support and around 646,000 people die from fall injuries. This situation is even more dangerous among elderly people. Frequently, elderly people are alone when a fall occurs, either at home or in other situations. Fall detection may allow decreasing the time until first aid is performed, reducing risks such as hypothermia, bronchopneumonia, dehydration, pressure sores and post-fall anxiety syndromes, which could compromise any Activity of Daily Living (ADL). Experimental data about using sensors to detect falls are encouraging. Sensors gather data to effectively detect and confirm falls, and, with a network connection, they can easily activate first aid and rescue procedures. Fall detection is being widely researched, and numerous fall detection systems have been created, but no specific method has been standardized or accepted globally [2]. This paper proposes a fall detection methodology and an application based on the use of five sensors: accelerometer, gyroscope, proximity sensor, microphone and GPS embedded in smartphones. This research targets all elderly people, not only those with limited mobility, but also those who want to use the application for prevention purposes, even if they are not currently concerned with fall events and have an independent lifestyle that includes outdoor activities.
For this reason, the proposed application does not include any technology installed in a specific location (i.e., ambient sensors) or wearable sensors. Indeed, elderly people, who does not have mobility problems, usually carry out activities alone and outdoor (for example going out to the grocery store). An elderly person rejects typically the idea of living by always wearing special devices that monitor her or his activities 24 hours a day; the smartphone is the type of device that could be mostly accepted because it is already part of people's daily lives. Back to the example, an elderly person could pick up her or his phone and go out. On the way to the grocery store, he could face an emergency, such as tripping over a manhole, and could have difficulty asking for help because she or he could be alone or unconscious. Furthermore, we had the intention of pursuing a limited data exchange between the device and the network both to make the system more efficient and to avoid privacy problems. Before detailing the description of the proposed application, we provide an analysis of the literature about fall detection. There are several studies discussing fall detection, as mentioned before. Fall detection systems can be grouped into the following categories: ambient-based, wearable and devices sensorbased, and hybrid that use a combination of both sensors (ambient and wearable) [3-5].

Ambient sensors used for fall detection mainly include floor sensors, microphones, infrared sensors, cameras and microwave motion detectors. Floor sensors are pressure sensors (piezoresistive 
or resistive) or a sensor pad/mat [6]. D Litvak, et al. [7] developed a fall detection system based on pattern recognition techniques using an accelerometer and microphones placed on the floor. The system could distinguish between a human or an object falling and Activity of Daily Living (ADL). Popescu, et al. [8] used a set of microphones consisting of a linear array of electret condenser acoustic sensors installed vertically to capture sound height information. Yun Li, et al. [9] proposed an acoustic system based on a circular microphone array and a data processing software that recognize the sound source and classify the sound as Fall or non-fall. Popescu, et al. [10] studied a system composed of four sets of two Passive InfraRed (PIR) sensors on vertical support. It takes advantage of measuring infrared light wavelengths: the human body has a specific measure of reflection, and a fall can be recognized by its specific pattern using a hidden Markov model. Ariani, et al. [11] used a wireless sensor network that emulates a Dual-Technology Sensor (DTS) motion detector, a hybrid sensor that combines PIR and microwave motion detectors and pressure mats.

Thome, et al. [12] used a camera approach, providing a theoretical analysis to define the optimal camera placement for detecting people falling in unspecified situations; they proved that two cameras are sufficient in practice. Fern'dez-Caballero, et al. [13] based their project on actuators and sensors (like an accelerometer and IR - InfraRed sensors) combined with image processing. Yu, et al. [14] proposed a detection system based on posture recognition using a single camera combined with a directed acyclic graph support vector machine for posture classification. Liang Liu, et al. [15] investigated different microwave sensor positions (floor, wall and ceiling) in two different experimental configurations. Many experiments made use of skin and wearable sensors with dedicated hardware, and recently, other experiments made use of smartphone sensors. Ren and Peng [6] have done a systematic analysis of fall detection and prevention technologies. Wearable and devices sensors (such as sensors embedded in smartphones) used for fall detection include accelerometers, gyroscopes, etc. Most research was focused on sensors applied directly to the skin or people's clothing/shoes. Bourke, et al. [16] presented eCAALYX, which is a wearable sensor system composed of health and mobility sensors; they use a one-axis accelerometer to detect a fall. Niazmand, et al. [17] presented a garment, a pullover "with integrated acceleration sensors, evaluation and control electronics." The system measures the acceleration of the torso and the arms. Sim, et al. [18] proposed an alternative position for the accelerometer: shoes. They calculate the change in acceleration values to recognize falls and ADLs Narasimhan, et al. [19] developed an adhesive sensor system composed of a tri-axial accelerometer, a microcontroller and a Bluetooth Low Energy transceiver, worn on a subject's torso. Park, et al. $[20,21]$ introduced a system composed of a 3-axis accelerometer, a 2-axis gyroscope, digital compasses or clinometer.
Tolkiehn, et al. [22] proposed a waist-worn sensor consisting of a 3-axis accelerometer and a barometric pressure sensor to detect a fall and its direction. Many research works used sensors already embedded in smartphones. He, et al. [23] proposed a solution with a waist-mounted smartphone that used the built-in accelerometer to detect falls. Lee, et al. [24] proposed a system using a tri-axial accelerometer embedded in a smartphone to distinguish fall events from ADLs considering the four directions of the falls (lateral, left and right, frontal, backwards). The measurements of variables returned by the sensors (usually one or two) indicate whether a fall event has happened or not. The results described in the cited works are obtained through experiments set in a laboratory. Ambient sensors are set up within a specific place/ambient, while the wearable/smartphone sensors collect and return data related to a specific person [25]. Another difference lies in the fact that ambient sensors are generally used indoors and are mainly for people that live in a controlled environment. In contrast, wearable/smartphone sensors are applicable both indoors and outdoors and can be used by people that have a more independent life. Wearable sensors have better results than sensors embedded into smartphones. However, people perceive skin and wearable sensors as foreign objects. Moreover, Kosse, et al. [26] observed that acceptance for fall detection sensor systems is not universal, using an analysis of some studies reporting positive cases. Other studies report somewhat mixed results in terms of incorporating sensor systems in care [27]. Smartphones are widely available; indeed, 3.5 billion people own smartphones in 2020 [28].

These devices are widely used and can be easily applied to detect falls. They can be used out of any clinical or wired environment, in any situation that is part of daily life and without any added equipment. Wearable sensors, in particular accelerometers (the most frequently used), applied to the skin usually perform better than sensors embedded in smartphones. Still, they are not practical and accepted because they are not integrated into the human body. The latest generation of smartphones is generally equipped with a variety of sensors such as an accelerometer, a gyroscope, a microphone, GPS and a proximity sensor. HawleyHague et al. suggest that acceptance of using sensors should be improved by making the usefulness of their adoption evident in terms of "...potential benefits such as independence, increased safety, convenience, increased social opportunities" [29]. The study underlines the users' need to maintain control over the use of technology, especially in regard to ambient sensors, which can cause more serious privacy issues. With these considerations and reflecting on the fact that most seniors lead an active life, this paper (as already explained before) focuses mainly on using smartphone sensors to collect data that indoor sensors cannot. Moreover, this research targets all elderly people who want to use the application for preventive purposes, even if they are not currently concerned with fall events. 
This paper starts with the idea of holistically using data collected by five sensors to detect and evaluate fall events and consequently activating first aid and rescue procedures. Indeed, it proposes the combined use of three sensors (accelerometer, gyroscope and microphone) to detect falls indoors and outdoors, the use of the GPS to determine the position of the subject in outdoor/indoor applications and the use of the proximity sensor to determine the state of the fallen person in order to organize first aid activities. There are two approaches to elaborate data gathered from the wearable/smartphone sensors for fall detection: threshold-based systems and machine learning-based systems [30,31]. Thresholdbased algorithms use a predefined fixed value to decide on a specific event; they require less computational power and are also less complex than other sophisticated algorithms [32]. The performance and accuracy of this kind of approach largely depend on threshold value tuning. Machine learning-based technologies produce results which can be considered similar to outcomes provided by threshold-based approaches [33,34]. Nevertheless, threshold-based algorithms have been popular because of their low computational overhead and complexity, while the machine learning approaches require high consumption of resources.

This paper describes the model and the software application for fall detection as it is defined and built. It takes advantage of five different sensors embedded in smartphones, removing the need to install ad-hoc sensors on the human body. This will result in wider acceptance of the application, as the target users already use the smartphone. In particular, the five smartphone sensors' data provide information that is useful for the detection and evaluation of probable fall events and, consequently, for activating the first aid process. All data are collected and computed on the smartphone; the communication flow is open only when a fall event is detected. This enables to minimize any privacy challenge. This work aims to contribute to the studies conducted so far by proposing a system that uses many different sensors embedded in smartphones and testing it on a range of types of falls, as explained in section III.

\section{The Main Contributions of this Paper are the following}

A. discussing how sensors built into a smartphone can be combined to detect and manage falls, without the need for adhoc hardware.

B. analyzing data acquired from different sensors and how to combine them.

\section{The Main Advantages of using Smartphone Sensors are the following}

A. They can be used by people from different ages that already have the device. Even if someone is not already interested in wearable sensors for fall detection, it can be used in the present or in the future for this scope.
B. The solution does not require any specific hardware (with related costs); any necessary hardware is already embedded in smartphones.

C. Beyond sensors, every smartphone provides an integrated communication channel; this supports and facilitates fast management of falls, notifying relatives and friends.

D. The solution is less invasive and more likely to be accepted because it does not modify peoples' habits.

E. Data can be managed and elaborated by the smartphone and it will be used to facilitate a rescue only if a fall is detected.

\section{The Use of Smartphones also has the following Disadvantages}

A. They have lower performance than body and wearable sensors. The few laboratory tests on smartphones' sensors report that applications based on smartphone sensors show performance about $15-20 \%$ lower than the ones based on body and wearable sensors.

B. They have variability in context conditions. Unlike body and wearable sensors, smartphone sensors face working conditions that can create variability in the detected values. Smartphones can be worn or carried in many different ways: at the belt, in the pocket, in hand, etc. These operative conditions influence the parameters used to detect falls, introducing uncertainty.

The previously cited disadvantages of using smartphones rather than wearable sensors have been mitigated by combining the inputs from five sensors embedded in a smartphone (fewer wearable sensors are generally used). The paper has the following organization. The next section will give the materials and methods used for detecting falls. It will describe the rescue process, including the system (based on PLAKSS - PLAtform for Knowledge and Services Sharing) components that is activated in case of falls. Section III describes the tests and their results; section IV provides a discussion by mapping experimental data and the three sensors' thresholds (accelerometer, gyroscope and microphone). Finally, section $\mathrm{V}$ concludes the paper.

\section{Materials and Methods}

As explained in the previous section, some literature studies describe systems that collect data from smartphone sensors, such as accelerometer or gyroscope. Aiming to mitigate the smartphone sensors performances weaknesses, this paper presents the results obtained using the fall detection process that we propose by combining data from smartphone sensors, in order to maximise the detection of fall events and, at the same time, reduce the problem of overfitting (i.e., filtering out false-positive), which could unnecessarily trigger phone calls and the rescue processes. 


\section{Method for Detecting Falls}

The process of detecting falls consists of two phases: detection and rescue, summarised in Figure 1. The detection phase has, as input, a real-time data flow coming from the smartphone's built-in sensors, i.e., the accelerometer, the gyroscope and the microphone. The algorithm analyses data flows to detect combinations of the three sensors' thresholds by comparing the fall matrix (see Section
IV). If the comparison detects a fall, the rescue phase is activated. In this phase, the system sends an emergency message to rescuers; this message also contains the data from the other two sensors, the GPS and the proximity sensor, to enrich the knowledge of the fall situation. The designed algorithm has been implemented in an Android app. The following subsections provide a detailed description of how the three sensors gather and process data flows.

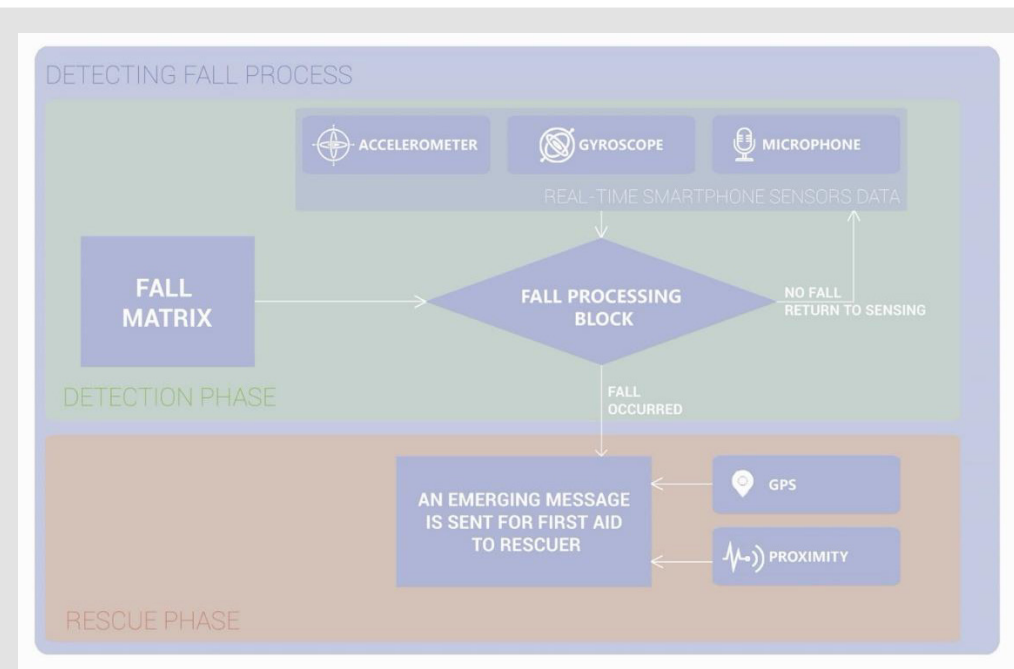

Figure 1: The process of fall detection and rescue.

Accelerometer: The accelerometer sensor returns values of acceleration along the three axes $\mathrm{x}, \mathrm{y}, \mathrm{z}$. It measures the acceleration Ad applied to the device, considering the gravity force $g$ and the other forces $\mathrm{F}$ that are applied to the sensor. The accelerometer's vertical dimension is calibrated to have a value equal to $9.8 \mathrm{~m} / \mathrm{s} 2$ to take gravity into account and measure acceleration correctly. This is the value provided by the sensor when the smartphone is motionless. Subsequently, the algorithm, developed on a smartphone app, calculates the Signal Magnitude Vector (SMV), also referred to as the Sum Vector (SV). In this way, the movement intensity is computed using this physical magnitude, depicted by equation (1).

$$
\text { Signal Magnitude Vector }=\sqrt{\left|A_{x}\right|^{2}+\left|A_{y}\right|^{2}+\left|A_{z}\right|^{2}}
$$

$A_{x}, A_{y}$ and $A_{z}$ represent signals according to the $x, y$ and $z$ components, respectively. We use the SMV to identify a probable fall event by deriving acceleration peak thresholds. Therefore, if a device is placed on a table with the screen facing upwards, it is possible to infer that:

a. Pushing the smartphone to the right causes a positive $\mathrm{x}$ acceleration value,

b. Pushing the smartphone forward causes a positive $y$ acceleration value,

c. Pushing the smartphone upwards with an acceleration, A, causes the $\mathrm{z}$ value of the acceleration to be equal to $\mathrm{A}+9.8 \mathrm{~m} /$ $\mathrm{s} 2$, because gravitational acceleration is also considered, d. When the device is in free Fall, it undergoes a rapid acceleration towards the ground of $9.8 \mathrm{~m} / \mathrm{s} 2$, and the accelerometer will measure an acceleration (2).

$$
g=0 \frac{m}{s^{2}}
$$

When a fall occurs, it is possible to observe that the acceleration suddenly decreases and then increases again, with a fast sequence of a peak close to zero (lower peak) and an upper peak in rapid succession (see Figure 2).

Several experiments have been done to identify the optimal threshold values: Figure 2 represents the average evolution of the SMV value, obtained during several tests. As already explained, a fall event is characterized by a rapid fluctuation of the SMV around the value of $9.8 \mathrm{~m} / \mathrm{s}^{2}$. The SMV suddenly decreases towards a minimum value and then increases towards a maximum value. Through peaks values analysis, we observed that the minimum value was in a range from 2 to $4 \mathrm{~m} / \mathrm{s} 2$ ", while the maximum value was in a range from 14 to $17 \mathrm{~m} / \mathrm{s}^{2}$. For this reason, we decided to use these experimentally obtained threshold values as input for the fall detection algorithm. Furthermore, it is possible to determine a timestamp (at around 1.5 seconds in Figure 2 associated with the beginning of the Fall based on the acceleration samples, in order to synchronize the samples collected from the different sensors. The fall time window starts 1.5 seconds before the timestamp mentioned above and ends 8.5 seconds after that (this time window includes four phases: free Fall, potential impact, post-fall and immobility). 


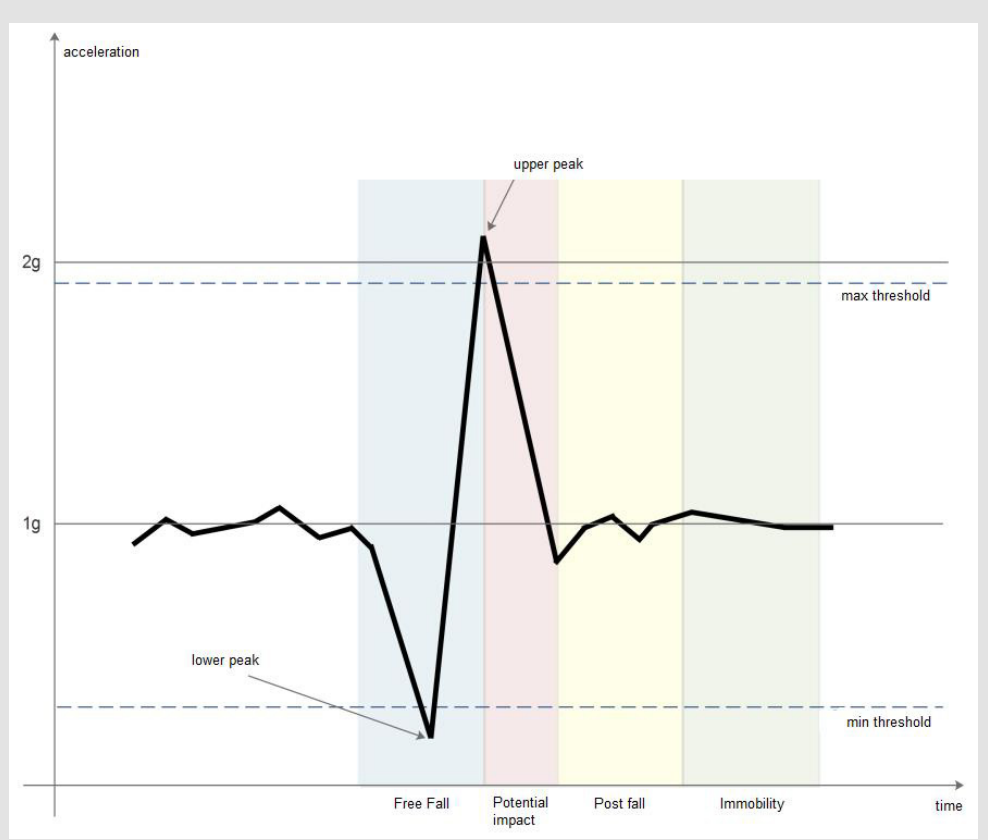

Figure 2: Evolution of the acceleration during a fall.

The samples frequency influences the precision in detecting threshold overcoming. High samples frequency detects all the peaks but causes more battery consumption; on the other hand, fewer samples frequency reduces battery consumption, but some peaks can be lost. Experiments and validations have been done to fix the optimal samples frequency: four different sample frequencies (fs $=1 / 5 ; 3 ; 6 ; 12$ [SPS]) have been considered and analyzed in the 10 seconds time frame around the potential Fall. After the experiments, we observed that the best option was six samples per second. The fall event is detected when the lower bound and the upper bound mentioned above are exceeded over the range of four consecutive samples. These values have been chosen to obtain the best compromise between acquiring the relevant information (that must not be lost) and lowering energy consumption due to data overhead. The flow chart, described in Figure 3, shows the algorithm used to detect acceleration features of an accidental fall. During the "Sampling" phase, the device performs periodic monitoring of the person's activity. Monitoring continues until a change in acceleration is observed, and in particular, until the acceleration value becomes lower than $\mathrm{S}_{\text {min }}$.

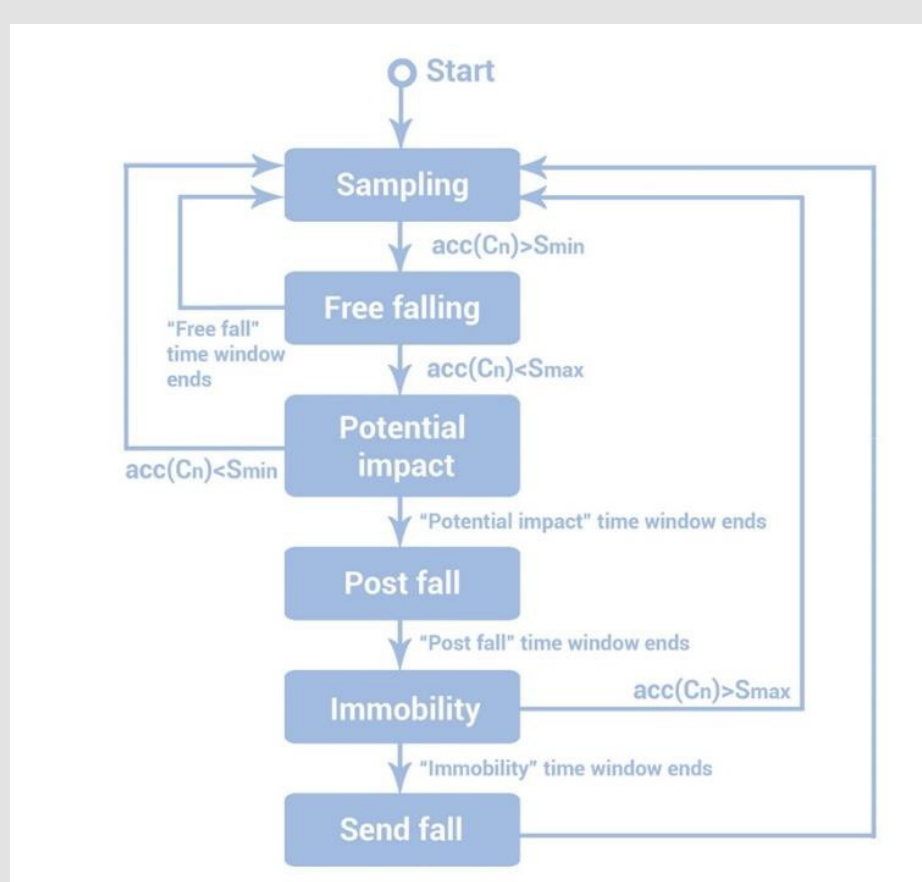

Figure 3: Flow-chart for detecting acceleration features that characterise an accidental fall. 
After the detection of an acceleration value lower than $\mathrm{S}_{\text {min' }}$, the relative time window begins (Free falling phase), and, during this timeslot, data streams are analyzed to determine if the characteristics of free Fall occur, i.e., if there is a decrease in acceleration up to a value close to zero, followed by a sudden increase in the acceleration value until it exceeds the threshold $\mathrm{S}_{\max }$. If the described sequence does not occur, the device returns to the "Sampling" state. Instead, if the observed acceleration values' characteristics are those of free Fall, the device goes into a state of "Potential impact". Then the "potential impact time window" begins. During this timeslot, a control starts to check if there are sudden changes in acceleration, which would indicate that the person is doing other activities (sitting, running, etc.). No checks are done on the threshold value $\mathrm{S}_{\max }$, because during the Fall this threshold might be exceeded several times: the person falling can hit the ground with different parts of his body at different times. Therefore, if the condition of an acceleration value greater than the minimum threshold occurs at the end of the post-fall time window, the state "Sending fall" is enabled. This means that the collected data will be sent to a server that will carry out further evaluations to confirm (or not) the Fall. To reduce the number of false alarms without increasing the linear computational effort, we decided to evaluate not only the accelerometer data but also data streams originated by the gyroscope and the microphone.

Gyroscope: The gyroscope sensor produces angular speed data along the three axes $\mathrm{x}, \mathrm{y}, \mathrm{z}$. These data are used in equation (3) to get the speed of rotation of a body:

$$
\omega=\sqrt{\left(\left|\omega_{x}\right|^{2}+\left|\omega_{y}\right|^{2}+\left|\omega_{z}\right|^{2}\right)}
$$

$\omega_{\mathrm{x}}{ }^{2}, \omega_{\mathrm{y}}{ }^{2}, \omega_{\mathrm{z}}^{2}$ are the angular speeds along the three axes $\mathrm{x}, \mathrm{y}, \mathrm{z}$, and the values are measured in degree/s. The fall event is observed by the algorithm based on acceleration samples and associated with a timestamp. To synchronize the data streams from the different sensors, samples from 1.5 seconds before the fall timestamp to 8.5 seconds after it are taken into account. During the Fall, the maximum value of the angular speed is detected. Two threshold values are considered. The first threshold value is associated with the first half of the 10 seconds interval in which the maximum angular velocity is detected. In the second half of the interval, if the Fall occurs, substantial immobility will be observed. The immobility implies that the angular velocity values should be lower than the minimum threshold. Through peaks values analysis, we observed that the minimum value was in a range 60 degree/s to 180 degree/s while the maximum value was in a range 200 degree/s to 320 degree/s. We decided to use these experimentally obtained threshold values to confirm the fall event.

Microphone: The microphone acquires the intensity of the noise produced during the Fall. The samples of the data acquired by the microphone are analyzed only if the accelerometer detects the features of a fall. To synchronize the samples collected from the different sensors, microphone samples from 1.5 seconds before the fall timestamp to 8.5 seconds after it are taken into account. The samples obtained during the experiments come from the microphone embedded in the smartphone. The Android APIs provide the MediaRecorder.getMaxAmplitude() method. This method returns the absolute value of a 16-bit integer (unsigned 16bit integer value) between 0 and 32767. These values cannot be related to any specific and calibrated physical measurement unit. Therefore, these values are only 16-bit digitization of an electrical output that varies between 0 and $100 \%$, where $100 \%$ corresponds to the maximum microphone voltage level. The values that this method reports are related to the sound pressure but differ from device to device. Therefore, the relative thresholds were derived experimentally using two different devices. It was possible to confirm that the output values generated belong to the same range in the case of these two devices. The values adopted belong to a reference scale from 0 to 12 , where 0 corresponds to the value 0 returned by the MediaRecorder.getMaxAmplitude() method, and 12 corresponds to 32400 . Each interval in the reference scale has a length of 2700 in terms of the values returned by the MediaRecorder. getMaxAmplitude() method.

Proximity Sensor: The proximity sensor collects samples of the proximity before, during, and after detecting the fall event. In the experimental tests, smartphones with binary (near, far) proximity sensors have been used.

GPS: The values of the coordinates are sent to the parents and first aid rescuers in order to locate the patient and allow assistance to be provided.

\section{The Rescue Processes}

In the case of confirmation of a fall, the system sends an alarm to family members and/or health assistants, and sends the patient's data to be rescued. GPS also sends the location of the fall event. The message also contains the state change of the smartphone proximity sensor, through which it is possible to determine whether the smartphone is close to the patient or not. Indeed, in the event of a fall, the proximity sensor can detect if the smartphone screen is in contact with some object; for example, a device could be in the person's pocket or bag (state $=1$ ), and as a consequence of the Fall the smartphone could come out and fall down. In this case, the display, at least for a short time, could change the state (from 1 to 0 ); this indicates that the patient may be far from the device after the Fall. Rescuers receive the emergency alert. They can call the patient to check her or his condition; if the person answers, she or he can directly provide information about the situation. Otherwise, it is assumed that the person is unable to answer, and this can imply that the person is in a state of unconsciousness or far from the device. 
The rescue process has the objective to provide rapid assistance to the fallen person. The network of a person's caregivers usually involves people (like relatives and friends) who live in close proximity to the patient who can provide first aid and assess the severity of the injuries.

The implementation of the rescue procedure required the configuration of a web platform with specific services. The rescue procedure is based on three main services:

a. The User-Handler is the service called for registration on the platform and for the login access to activate the service. Each person can disable the service based on her or his preferences.

b. The Emergency-Handler is the service called when 30 seconds have passed, after a fall has been detected, without any input from the user (see Figure 4) or when the user presses the
SOS button (see Figure 2). This service sends a message to a relative's/friend's or a caregiver's smartphone through the Google Messaging Cloud (GMC) system containing the information related to the patient and type of emergency.

\section{The System in Action}

On the patient side, i.e., on the smartphone, an app continuously runs in the background after a login procedure, gaining data from the sensors. Figure 4 shows different screenshots of the smartphone app. The login page (a) enables the activation (b) of the continuous monitoring (c) If a Fall occurs, the app shows an alarm page with red and green buttons (d) where the user can confirm or disable the alarm. If the user confirms the emergency request or after 30 seconds, the app shows the sent emergency page (e) As represented in Figure 5, there are four possibilities:

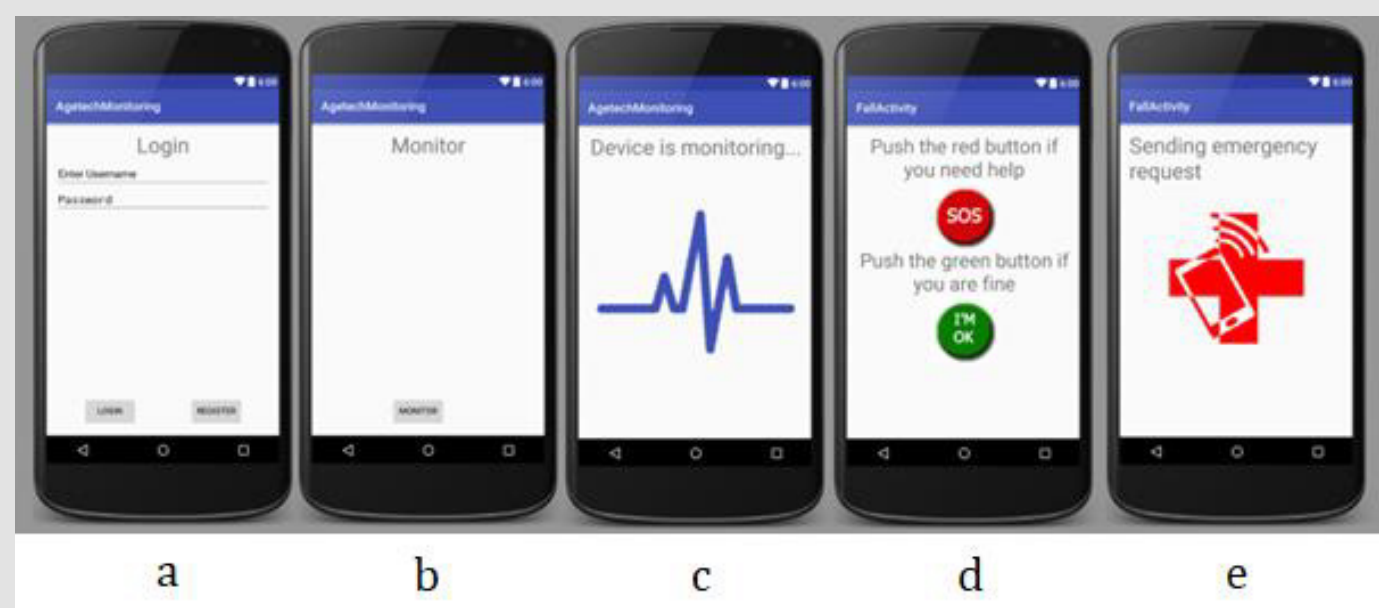

Figure 4: Different screenshots of the app on the smartphone.

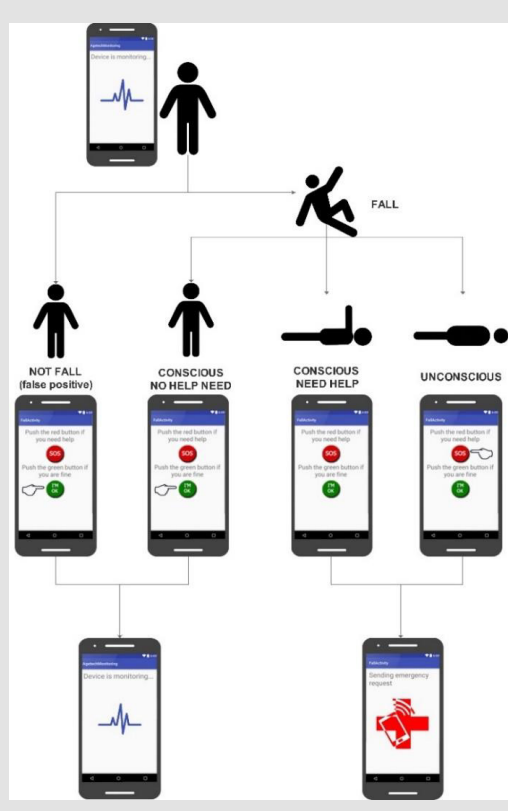

Figure 5: Different app states during monitoring and fall detection. 
a. The patient has fallen and is unconscious-the smartphone displays the buttons on the screen, emitting the emergency sound. After 30 seconds without any interaction, it sends an alarm to the caregiver's (relatives, doctors, etc.) smartphone. This message contains the GPS position, the fallen person's data and the change of state of the proximity sensor, through which it is possible to infer whether or not the smartphone is near the patient.

b. The patient fell; she or he is conscious and needs help-the smartphone displays the buttons on the screen, emitting the emergency sound. If the patient presses the red button, the device immediately sends an alarm to the caregiver's smartphone. As described above, the message contains GPS position, patient data and the state of the proximity sensor.

c. The person has not fallen, but the device detects a fall event (false positive). In this case, the patient can press the green button to disable the emergency procedure.

d. The person has fallen, but she or he does not need any kind of assistance. In this case, the patient can press the green button to disable the emergency procedure.

e. Depending on the user's actions (or if there is no action), the system will change the state from Alarm to Listening or Management.

\section{Results}

The application acquires and analyses three streams of data on the smartphones for assessing a fall: the streams of the accelerometer, gyroscope and microphone (GPS and proximity sensor are used to support the first aid and rescue phase). If a fall is detected, a rescue procedure is activated. The first aid request is the only case of data transmission to the server. Indeed, the proposed methodology avoids to send all the data stream in a continuous cycle: this choice has been made to limit battery consumption and privacy issues. It has been demonstrated that "a single accelerometer sensor at $200 \mathrm{~Hz}$ generates about $2.3 \mathrm{~GB}$ of data per day, the more the sensors or monitoring metrics are added, the more the data are generated by the sensors" [35]. If all these data have to be uploaded to the cloud for analytics or sent to a server as an input for machine learning approaches, it would result in a wastage of network bandwidth, high consumption of the smartphone battery, a decrease in response time and efficiency and a continuous activity monitoring that lead to user's privacy deprivation.

\section{Experimental Setting}

Falls are very relevant from the epidemiological point of view due to their consequences (the fifth leading cause of death); however, fall events are quite rare and difficult to detect in a real-life environment. Consequently, it is difficult to collect real data. Only some studies collected fall data in real-life environments in order to analyze the acceleration values during falls. The work of Bai, et al. [25] brought out differences between real and simulated falls and described how to obtain, in laboratory tests, simulated fall data comparable to real fall data. For this reason, our laboratory tests have been set up, taking into account the studies and the results described in [25]. The steps followed for setting up the experiments are listed below:

a. The equipment chosen for the experiments includes five sensors built into smartphones (accelerometer, gyroscope, proximity sensor, microphone and GPS) that acquire and gather data, which, when properly processed, can be useful for detecting and assessing a fall (Figure 6). Such sensors have different operating conditions, roles and relevance when detecting and assessing a fall.

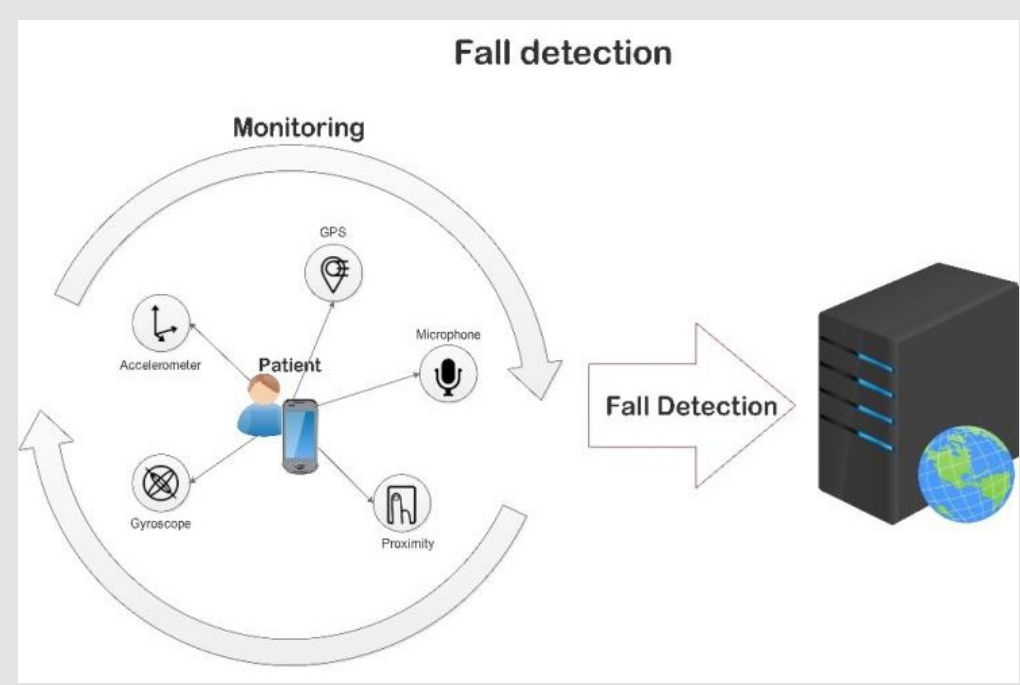

Figure 6: Smartphone sensors useful in a fall detection process. 
b. We considered two possible positions for the smartphone: in a pocket or using a support hooked to a belt. Twenty-five people with various characteristics were selected to participate in the experiment. There were twelve women and thirteen men with various weights, and ages from 20 to 45 years. The data samples were collected from healthy subjects as experiments of simulated falls by senior citizens may be improper and dangerous. This setting is conventional in literature; it could not reflect senior citizens' activities, but it represents the first step before testing the application on a large scale and everyday life that can require a very long period (one or more years). Data samples from senior and in real life need to be investigated in the future to identify any potential discrepancies.

c. 100 tests per person have been conducted, divided into 50 falls and 50 ADL events.

d. The activity of the initial setting of the parameters (variables related to each sensor) for detecting falls took into account the experiments of Klenk, et al. [36].

e. Preliminary data and literature data allowed us to adjust the parameters.

The tests involved twenty-five people simulating 50 falls (on mats) each-one, with 1250 falls in total. The types of performed falls aim, as much as possible, to simulate the most common falls occurring in the elderly population. Lord et al. claim that $82 \%$ of falls occur from a standing position. Therefore, the falls examined explicitly during this phase were originated from an upright position and continued in all directions (forward, backward, lateral).

The types of falls analyzed are detailed below:

a. Forward: A fall in the direction of walking, causing an impact on the pelvis.

b. Backwards: A fall due to a slip, opposite to the direction of walking, causing an impact on the back.

c. Lateral: A fall due to simulated fainting, collapsing on legs, causing an impact on the hip.

d. Forward on knees: A fall in the direction of walking, causing an impact on the knees.

At the same time, participants were also asked to carry out some daily activities (ADL) to produce inputs with values similar to those collected when a fall occurs, creating false alarms. Some examples of these activities are sitting on a sofa, lying down on a bed and running up the stairs. 1250 daily activities have been collected, or 50 for each person participating. In these experiments, people had the smartphone in their pocket.

\section{Experimental Data}

The tests addressed the necessity of identifying optimal settings of the data detected by the sensors for different smartphones, with the aim of maximizing sensitivity, specificity and accuracy. The data collected concern all five sensors, but a specific analysis has been done on the accelerometer, the gyroscope and the microphone to set the best operative parameters related to the fall detection phase. This enabled us to establish the most suitable trade-off between minimum and maximum threshold variation. This subsection illustrates the data collected from the accelerometer, gyroscope and microphone, considering different possible thresholds and different classification values (i.e. True Negative, False Negative, True Positive, False Positive), and evaluating sensitivity, specificity and accuracy. The data collected are analyzed to understand which combinations of thresholds are associated with fall detections, and which combinations are associated with ADL.

Data and Thresholds: The first objective is to select the threshold values that activate the process of fall evaluation. From Tables $1 \& 2$, it is possible to observe how incrementing the maximum threshold (keeping the minimum constant) decreases the sensitivity but increases the specificity. Analogously, if the minimum threshold is decremented, the sensitivity decreases and the specificity increases. The impact on the practical use of the fall detector is that if the settings prioritize the objective to detect any fall, it is necessary to manage many false falls. When choosing the threshold values, it is also necessary to consider that a fall event is relatively rare; at the same time, false alarms produced by daily activities can be quite frequent. On the contrary, if the settings prioritize reducing false alarms, the system detects a smaller percentage of true falls.

Table 1: Variables analysed for threshold variation for the accelerometer, the gyroscope and the microphone.

\begin{tabular}{|c|c|}
\hline Variable & Explanation \\
\hline $\mathrm{TN}=$ True Negative & $\begin{array}{l}\text { Number of false instances } \\
\text { classified as false. }\end{array}$ \\
\hline FN $=$ False Negative & $\begin{array}{l}\text { Number of true instances classified } \\
\text { as false. }\end{array}$ \\
\hline $\mathrm{TP}=$ True Positive & $\begin{array}{l}\text { Number of true instances classified } \\
\text { as true. }\end{array}$ \\
\hline FP $=$ False Positive & $\begin{array}{l}\text { Number of false instances } \\
\text { classified as true. }\end{array}$ \\
\hline Sensitivity $=[\mathrm{TP} /(\mathrm{TP}+\mathrm{FN})] * 100$ & $\begin{array}{l}\text { Percentage of true correctly } \\
\text { classified with respect to the total } \\
\text { true instances. }\end{array}$ \\
\hline Specificity $=[\mathrm{TN} /(\mathrm{TN}+\mathrm{FP})] * 100$ & $\begin{array}{l}\text { Percentage of false correctly } \\
\text { classified with respect to the total } \\
\text { false instances. }\end{array}$ \\
\hline $\begin{array}{c}\text { Accuracy }=[(\mathrm{TP}+\mathrm{TN}) / \text { total } \\
\text { instances }] * 100\end{array}$ & $\begin{array}{l}\text { Percentage of true and false } \\
\text { correctly classified for the total } \\
\text { instances. }\end{array}$ \\
\hline
\end{tabular}

Accelerometer: The first set of experiments allowed us to establish that better values are obtained with a minimum threshold value of around $3 \mathrm{~m} / \mathrm{s} 2$ and a maximum threshold value of about $15 \mathrm{~m} / \mathrm{s}^{2}$ (see Figure 2). For this reason, the tests were conducted with minimum threshold values of about $3 \mathrm{~m} / \mathrm{s} 2$ (range 2-4) and 
maximum threshold values of about $15 \mathrm{~m} / \mathrm{s}^{2}$ (range 14-17). Tables 2 \& 3 summarize the results obtained using different threshold values for the accelerometer. The data show that lowering the minimum threshold produces a loss of sensitivity, i.e., fewer falls are detected (false negatives are present). When the threshold is raised, more falls are detected, but many false positives are generated.
On the other hand, high values of the maximum threshold cause a decrease in sensitivity and an increase in specificity. Decreasing the maximum threshold value causes the sensitivity to increase, i.e., more falls are detected. At the same time, however, specificity decreases, i.e., more false positives are generated.

Table 2: Classification values for different thresholds with an accelerometer.

\begin{tabular}{|c|c|c|c|c|}
\hline THRESHOLD VALUE & TN & FN & TP & FP \\
\hline $\mathrm{MAX}=14 \mathrm{MIN}=2$ & 819 & 406 & 844 & 431 \\
\hline $\mathrm{MAX}=14 \mathrm{MIN}=3$ & 813 & 69 & 1181 & 437 \\
\hline MAX $=14$ MIN=4 & 550 & 19 & 1231 & 700 \\
\hline $\mathrm{MAX}=15 \mathrm{MIN}=2$ & 1100 & 631 & 619 & 150 \\
\hline MAX $=15$ MIN $=3$ & 1082 & 112 & 1138 & 168 \\
\hline MAX $=15$ MIN $=4$ & 881 & 31 & 1219 & 369 \\
\hline $\mathrm{MAX}=16 \mathrm{MIN}=2$ & 1131 & 656 & 594 & 119 \\
\hline $\mathrm{MAX}=16 \mathrm{MIN}=3$ & 1107 & 287 & 963 & 143 \\
\hline $\mathrm{MAX}=16 \mathrm{MIN}=4$ & 926 & 212 & 1038 & 324 \\
\hline$M A X=17$ MIN $=2$ & - & - & - & - \\
\hline$M A X=17$ MIN=3 & 1219 & 500 & 750 & 31 \\
\hline MAX $=17$ MIN $=4$ & - & - & - & - \\
\hline
\end{tabular}

Table 3: Measured values of sensitivity, specificity and accuracy for different values of the thresholds.

\begin{tabular}{|c|c|c|c|}
\hline THRESHOLD VALUE & Sensitivity & Specificity & Accuracy \\
\hline $\mathrm{MAX}=14 \mathrm{MIN}=2$ & $67.52 \%$ & $65.52 \%$ & $66.52 \%$ \\
\hline $\mathrm{MAX}=14 \mathrm{MIN}=3$ & $94.48 \%$ & $65.04 \%$ & $79.76 \%$ \\
\hline $\mathrm{MAX}=14 \mathrm{MIN}=4$ & $98.48 \%$ & $44.00 \%$ & $71.24 \%$ \\
\hline $\mathrm{MAX}=15 \mathrm{MIN}=2$ & $49.52 \%$ & $88.00 \%$ & $68.76 \%$ \\
\hline $\mathrm{MAX}=15 \mathrm{MIN}=3$ & $91.04 \%$ & $86.56 \%$ & $88.80 \%$ \\
\hline $\mathrm{MAX}=15 \mathrm{MIN}=4$ & $97.52 \%$ & $70.48 \%$ & $84.00 \%$ \\
\hline $\mathrm{MAX}=16 \mathrm{MIN}=2$ & $47.50 \%$ & $90.48 \%$ & $69.00 \%$ \\
\hline $\mathrm{MAX}=16 \mathrm{MIN}=3$ & $77.04 \%$ & $88.56 \%$ & $82.80 \%$ \\
\hline $\mathrm{MAX}=16 \mathrm{MIN}=4$ & $83.04 \%$ & $74.08 \%$ & $78.56 \%$ \\
\hline $\mathrm{MAX}=17 \mathrm{MIN}=2$ & - & - & - \\
\hline $\mathrm{MAX}=17 \mathrm{MIN}=3$ & $60.00 \%$ & $97.52 \%$ & $78.76 \%$ \\
\hline $\mathrm{MAX}=17 \mathrm{MIN}=4$ & - & - & - \\
\hline
\end{tabular}

Gyroscope: The experiments performed have shown that the samples taken during the 10 second window can be divided into two temporal windows that can be associated with the first phase of a fall (which also includes the sample related to the fall event observed by the algorithm) and the second phase, i.e., the post phase of the Fall. In the first phase, the first 150 samples are analyzed to extract the maximum value. During this phase, a sample must have an angular speed up to the maximum threshold. During the second phase, it is assumed that the patient is motionless on the ground, and the angular speed is lower than a threshold value; if this condition is verified, the data gathered from the gyroscope confirm the fall event. Also, in this case, if we set the gyroscope to detect any fall, it is necessary to manage many false falls. It is also essential to consider in the choice of the threshold values (similarly to the accelerometer) that a fall event is relatively rare, while normal activities that could create false alarms are quite frequent. On the contrary, if we set the gyroscope to reduce false alarms, the system detects a smaller percentage of true falls. Tables $4 \& 5$ display the results obtained using different threshold values for the gyroscope. 
Table 4: Classification values obtained with a gyroscope for different thresholds.

\begin{tabular}{|c|c|c|c|c|}
\hline THRESHOLD VALUE & TN & FN & TP & FP \\
\hline $\mathrm{MAX}=200 \mathrm{MIN}=60$ & 932 & 521 & 729 & 318 \\
\hline $\mathrm{MAX}=200 \mathrm{MIN}=120$ & 870 & 471 & 779 & 380 \\
\hline $\mathrm{MAX}=200 \mathrm{MIN}=180$ & 700 & 415 & 835 & 550 \\
\hline $\mathrm{MAX}=260 \mathrm{MIN}=60$ & 970 & 810 & 440 & 280 \\
\hline $\mathrm{MAX}=260 \mathrm{MIN}=120$ & 907 & 759 & 491 & 343 \\
\hline $\mathrm{MAX}=260 \mathrm{MIN}=180$ & 801 & 709 & 541 & 450 \\
\hline$M A X=320 \mathrm{MIN}=60$ & 1001 & 1079 & 171 & 249 \\
\hline $\mathrm{MAX}=320 \mathrm{MIN}=120$ & 945 & 1041 & 209 & 305 \\
\hline $\mathrm{MAX}=320 \mathrm{MIN}=180$ & 882 & 1004 & 246 & 368 \\
\hline $\mathrm{MAX}=340 \mathrm{MIN}=60$ & 1026 & 1079 & 171 & 224 \\
\hline $\mathrm{MAX}=340 \mathrm{MIN}=120$ & 970 & 1041 & 209 & 280 \\
\hline $\mathrm{MAX}=340 \mathrm{MIN}=180$ & 914 & 1004 & 246 & 336 \\
\hline
\end{tabular}

Table 5: Measured values of sensitivity, specificity and accuracy for different values of the thresholds with a gyroscope.

\begin{tabular}{|c|c|c|c|}
\hline THRESHOLD VALUE & Sensitivity & Specificity & Accuracy \\
\hline MAX=200 MIN=60 & $58.32 \%$ & $74.56 \%$ & $66.44 \%$ \\
\hline$M A X=200 M I N=120$ & $62.32 \%$ & $69.60 \%$ & $65.96 \%$ \\
\hline$M A X=200 M I N=180$ & $66.80 \%$ & $56.00 \%$ & $61.40 \%$ \\
\hline$M A X=260 M I N=60$ & $35.20 \%$ & $77.60 \%$ & $56.40 \%$ \\
\hline$M A X=260 M I N=120$ & $39.28 \%$ & $72.56 \%$ & $55.92 \%$ \\
\hline$M A X=260 M I N=180$ & $43.28 \%$ & $64.08 \%$ & $53.68 \%$ \\
\hline$M A X=320 M I N=60$ & $13.68 \%$ & $80.08 \%$ & $46.88 \%$ \\
\hline$M A X=320 M I N=120$ & $16.72 \%$ & $75.60 \%$ & $46.16 \%$ \\
\hline$M A X=320 M I N=180$ & $19.68 \%$ & $70.56 \%$ & $45.12 \%$ \\
\hline$M A X=340 M I N=60$ & $13.68 \%$ & $82.08 \%$ & $47.88 \%$ \\
\hline$M A X=340 M I N=120$ & $16.72 \%$ & $77.60 \%$ & $47.16 \%$ \\
\hline$M A X=340 M I N=180$ & $19.68 \%$ & $73.12 \%$ & $46.40 \%$ \\
\hline
\end{tabular}

Microphone: The experiments on the microphone have been conducted using six threshold values (from 6 to 11). In order to evaluate microphone data, sensitivity, specificity, and accuracy have been calculated for each threshold's value. When choosing threshold values, it is necessary to consider that although a fall event is relatively rare, everyday activities that could create false alarms are quite frequent. Analysis of real falls shows that an average, noisy room can provide amplitude values of sound ranging between 2-4, a very noisy room between 5-8. A very loud sound is found to have a value greater than 9 . The results prove that choosing the threshold's value between 6-8 requires managing a more significant number of false positives. On the contrary, setting the threshold's value greater than 10 causes the number of false negatives growing dramatically. This proves that with these threshold values, the system cuts off real falls. Tables 6 \& 7 show the results obtained using different threshold values for the microphone. The threshold values considered in the experiment are those in which the specificity is over $40 \%$, and the sensitivity is around $90 \%$.

Table 6: Classification values obtained for different thresholds with a microphone.

\begin{tabular}{|c|c|c|c|c|}
\hline THRESHOLD VALUE & TN & FN & TP & FP \\
\hline 6 & 337 & 45 & 1205 & 913 \\
\hline 7 & 375 & 51 & 1099 & 875 \\
\hline 8 & 444 & 70 & 1180 & 806 \\
\hline 9 & 550 & 95 & 1155 & 700 \\
\hline 10 & 675 & 120 & 1130 & 575 \\
\hline
\end{tabular}


Table 7: Values of sensitivity, specificity and accuracy for different values of the thresholds with a microphone.

\begin{tabular}{|c|c|c|c|}
\hline THRESHOLD VALUE & Sensitivity & Specificity & Accuracy \\
\hline 6 & $96.40 \%$ & $26.96 \%$ & $61.68 \%$ \\
\hline 7 & $87.92 \%$ & $30.00 \%$ & $58.96 \%$ \\
\hline 8 & $94.40 \%$ & $35.52 \%$ & $64.96 \%$ \\
\hline 9 & $92.40 \%$ & $44.00 \%$ & $68.20 \%$ \\
\hline
\end{tabular}

Proximity Sensor: The experiments carried out show that the samples from this sensor usually are not guaranteed to detect the Fall, except for in the (rare) case of a change in the state of the proximity sensor. It can provide additional information concerning data from other sensors. Indeed, we decided to keep this information about the probable positioning of the smartphone after the Fall. If the patient wears the mobile phone on his side, using a support hooked to his belt, the proximity sensor state usually remains unchanged. Experiments have shown that if the smartphone disengages from the belt support due to the impact of a fall, the value measured by this sensor can have one or more variations. If the smartphone is usually contained in the person's pocket or bag, it could come out of the person's pocket or bag as a consequence of a fall, and the display (at least for a short time) may not be in contact with any object (pocket, bag). This behavior can change with different persons. This information is also significant at the time of the rescue because the patient may be conscious but may not answer the phone because of her or his distance from the device. In the case of a change of state of the proximity sensor, a set of less restrictive thresholds is taken into consideration.

\section{Discussion}

This section provides a discussion by mapping experimental data and the accelerometer, gyroscope, and microphone thresholds. The thresholds considered in the accelerometer, gyroscope, and microphone tests identify $864(12 * 12 * 6)$ possible combinations of values associated with these three sensors. Based on the results of these tests, only 84 combinations are very relevant for detecting falls, with a sensitivity of $98 \%$ of real falls. These 84 combinations $\left(7^{*} 6 * 2\right)$ are associated with the thresholds in Tables $2,4 \& 7$. The detection of true falls is very satisfying; however, these combinations caused an unsatisfyingly high number of detections of false positives (ADL events detected as falls), with a value of specificity of $83.04 \%$. Considering the frequency of the ADLs, it is necessary to increase the specificity value to avoid an excessive number of false alarms. For this reason, starting from the 84 combinations selected, the experimental results allowed us to select the map of combinations that will give acceptable specificity values. The minimum value considered acceptable was $92 \%$. The experimental data have been analyzed to select the best map of combinations to acquire the acceptable value of specificity and at the same time, to avoid losing, as much as possible, sensitivity in detecting real falls. The map of combinations shown in Table 8 has been used as an input for the fall detection system, and with several tests, in the same conditions described above, we obtained a sensitivity value of $89.28 \%$.

Table 8: Map of the combinations chosen for optimising specificity and sensitivity.

\begin{tabular}{|c|c|c|c|c|c|c|}
\hline $\begin{array}{l}\text { Microphone } \\
\text { Threshold=9 }\end{array}$ & \multicolumn{6}{|c|}{ Gyroscope } \\
\hline Accelerometer & $\mathrm{MAX}=200 \mathrm{MIN}=60$ & $\begin{array}{l}M A X=200 \\
M I N=120\end{array}$ & $\begin{array}{l}M A X=200 \\
M I N=180\end{array}$ & $\mathrm{MAX}=260 \mathrm{MIN}=60$ & $\begin{array}{l}M A X=260 \\
M I N=120\end{array}$ & $\begin{array}{l}M A X=260 \\
M I N=180\end{array}$ \\
\hline \multicolumn{7}{|l|}{ MAX $=14$ MIN=3 } \\
\hline \multicolumn{7}{|l|}{$\mathrm{MAX}=14 \mathrm{MIN}=4$} \\
\hline \multicolumn{7}{|l|}{ MAX=15 MIN=3 } \\
\hline \multicolumn{7}{|l|}{$\mathrm{MAX}=15 \mathrm{MIN}=4$} \\
\hline \multicolumn{7}{|l|}{ MAX $=16$ MIN=3 } \\
\hline \multicolumn{7}{|l|}{$\mathrm{MAX}=16 \mathrm{MIN}=4$} \\
\hline \multicolumn{7}{|l|}{ MAX $=17$ MIN=3 } \\
\hline $\begin{array}{c}\text { Microphone } \\
\text { Threshold=10 }\end{array}$ & \multicolumn{6}{|c|}{ Gyroscope } \\
\hline Accelerometer & $\mathrm{MAX}=200 \mathrm{MIN}=60$ & $\begin{array}{l}M A X=200 \\
M I N=120\end{array}$ & $\begin{array}{l}M A X=200 \\
M I N=180\end{array}$ & $\mathrm{MAX}=260 \mathrm{MIN}=60$ & $\begin{array}{l}M A X=260 \\
M I N=120\end{array}$ & $\begin{array}{l}M A X=260 \\
M I N=180\end{array}$ \\
\hline $\mathrm{MAX}=14 \mathrm{MIN}=4$ & & & & & & \\
\hline $\mathrm{MAX}=15 \mathrm{MIN}=3$ & & & & & & \\
\hline $\mathrm{MAX}=15 \mathrm{MIN}=4$ & & & & & & \\
\hline
\end{tabular}




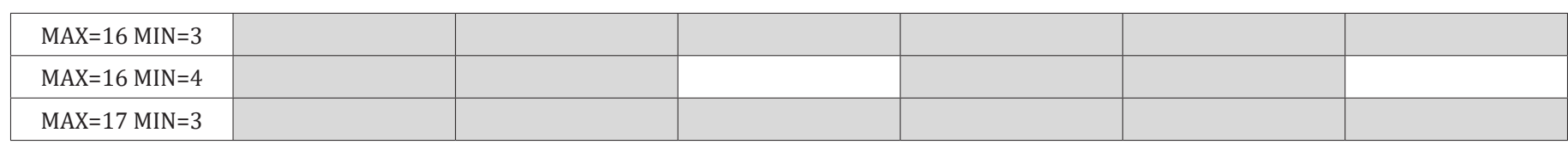

Table 9: Map of the combinations chosen for optimising specificity and sensitivity.

\begin{tabular}{|c|c|c|}
\hline Method & Specificity & Sensitivity \\
\hline [25] & $\mathrm{N} / \mathrm{A}$ & $\mathrm{N} / \mathrm{A}$ \\
\hline [22] & $85.24 \%$ & $87.77 \%$ \\
\hline [23] & $\mathrm{N} / \mathrm{A}$ & $\mathrm{N} / \mathrm{A}$ \\
\hline [21] & $\mathrm{N} / \mathrm{A}$ & N/A \\
\hline [33] & $88.57 \%$ & $89.20 \%$ \\
\hline Our approach & $92 \%$ & $89.28 \%$ \\
\hline
\end{tabular}

The way that we have chosen the combinations in this paper is only the first step. Wider experimentation on the field, with a large number of users will enable to refine finding the optimal combinations. It is important to set up combinations of threshold values representing the best solution for sensitivity and specificity. Another goal is to create different settings of the cloud of thresholds based on users' features such as weight, age and gender. Then, the cloud of values could be adapted based on individually collected data. Table 9 compares performances of our methodology with [21-23,25,33]. As shown in the following table, not all the cited works show measurable results. As compared to [22], our approach proposes a more practical system, avoiding additional sensors to be worn by the user, which only consists in a smartphone, achieving better results in terms of specificity and sensitivity. In the same way in [33], the system consists in a wearable device to be worn at the waist which detects Fall through the use of a gyroscope and an accelerometer: analyzing its results, it can be observed that our approach is pretty similar in terms of sensitivity, however it can achieve better results in detecting true negatives (with a variation of about $3.5 \%$ in specificity).

\section{Conclusion}

In this study, we developed a fall detection system for elderly people using smartphones. Our initial hypothesis was to design a fall detection system that is not perceived as invasive, widely accepted by people, and based on already existing and used technologies. Therefore, we decided to design the application based on smartphone sensors to make it available to as many people as possible. Indeed, using a smartphone, people do not have to wear other devices or sensors; this is a strength for the acceptance by the elderly. We developed a fall detection algorithm that uses data streams from five sensors (accelerometer, gyroscope, microphone, proximity and GPS) embedded in a smartphone. The algorithm works with thresholds and time windows. We demonstrated that our algorithm can work efficiently using ranges of thresholds experimentally defined; it recognizes falls through the combination of these ranges of values and streams from gyroscope and accelerometer, with further refinements done by microphone and proximity sensor. Our system can be used during outdoor activities by elderly people, for example. The smartphone, which runs the proposed system, monitors user movements through its sensors. If a fall occurs, the system recognizes specific conditions, using thresholds and time windows, and sends an alarm to the server which forwards the first aid request to pre-selected rescuers. As already explained, an essential hypothesis that we assumed was to respond to the privacy issues and to build a non-invasive system. To achieve these objectives, we limited data exchange, using a server only for the rescue management procedure; we investigated on how to reduce the false positives to avoid many false alarms which could induce users to stop using the system. The algorithm has been proven to have good performance in terms of sensitivity, specificity, accuracy and computational complexity. The algorithm takes about 6.5 seconds to detect the Fall and collect the necessary data to be sent remotely. The entire system takes, on average, less than 1 minute to ensure that the emergency notification arrives on the smartphone of the person responsible for the rescue; these values can vary depending on the traffic conditions in the communication network.

For future work, many issues need to be further investigated. Our algorithm is going to be tested through realistic experiments, in a similar way described in [24]: we planned to distribute the application on a wider scale to verify its efficacy in everyday life activities. This large scale and testing phase will have a duration of two years. We planned to test the entire system in real operating conditions involving many people, including people whose age is greater than the ones we tested on to produce this work. The low cost facilitates the activation of the experimentation. The experiments will use devices (smartphones) that are already available for the population. The testing phase in the field will enable both tunings of optimal parameters and the extension of the trial over the devices considered during the test phase. Finally, regarding emergency management, the combination of IoT (Internet of Things) and communication channels can enlarge the perspective of safety, and healthcare in the future toward a social paradigm, in which smart objects participate as active agents [36- 
38] in collaborative social networks with people (patients, relatives, friends and caregivers).This is particularly relevant to senior citizens living in their homes [39] or that have an independent life. It allows early detection of a fall, a fast verification of the real conditions and integrated management of potential emergencies based on proximity (involving relatives and friends), competence and knowledge[40].

\section{References}

1. (2020) World Health Organization. Falls, WHO.

2. C Tacconi, S Mellone, L Chiari (2011) Smartphone-based applications for investigating falls and mobility, in Proceedings of the $5^{\text {th }}$ International Conference on Pervasive Computing Technologies for Healthcare (Pervasive Health), Dublin, Ireland pp. 258-261.

3. A Ramachandran, A Karuppiah (2020) A survey on recent advances in wearable fall detection systems. in BioMed Research International $\mathrm{p}$. $1-17$.

4. Nizam Y, Jamil MMA (2020) Classification of Daily Life Activities for Human Fall Detection: A Systematic Review of the Techniques and Approaches. In: Ponce H, Martínez Villaseñor L, Brieva J, Moya Albor E (Eds.)., Challenges and Trends in Multimodal Fall Detection for Healthcare. Studies in Systems, Decision and Control, Springer, 273.

5. Singh SU Rehman, S Yongchareon, PHJ Chong (2020) Sensor Technologies for Fall Detection Systems: A Review. In IEEE Sensors Journal 20(13): 6889-6919.

6. L Ren, Y Peng (2019) Research of fall detection and fall prevention technologies: A systematic review. In IEEE Access 7(201): 77702-77722.

7. Y Zigel, D Litvak, I Gannot (2009) A method for automatic fall detection of elderly people using floor vibrations and sound-Proof of concept on human mimicking doll falls. In IEEE Transactions on Biomedical Engineering 56(12): 2858-2867.

8. M Popescu, Y Li, M Skubic, M Rantz (2008) An acoustic fall detector system that uses sound height information to reduce the false alarm rate. In 2008 30th Annual International Conference of the IEEE Engineering in Medicine and Biology Society, Vancouver, BC pp. 4628-4631.

9. Y Li, KC Ho, M Popescu (2012) A microphone array system for automatic fall detection. In IEEE Transactions on Biomedical Engineering 59(5): 1291-1301.

10. M Popescu, B Hotrabhavananda, M Moore, M Skubic (2012) VAMPIR- an automatic fall detection system using a vertical PIR sensor array. In 2012 6th International Conference on Pervasive Computing Technologies for Healthcare (PervasiveHealth) and Workshops, San Diego, CA, pp. 163166.

11. A Ariani, SJ Redmond, D Chang, NH Lovell (2012) Simulated unobtrusive falls detection with multiple persons. In IEEE Transactions on Biomedical Engineering 59(11): 3185-3196.

12. N Thome, S Miguet, S Ambellouis (2008) A real-time, multiview fall detection system: A LHMM-based approach. In IEEE Transactions on Circuits and Systems for Video Technology 18(11): 1522-1532.

13. A Fernndez Caballer, Marina V, Juan SC, Jose C, Veronica M, et al (2012) HOLDS: Efficient fall detection through accelerometers and computer vision. In 2012 Eighth International Conference on Intelligent Environments, Guanajuato pp. 367-370.

14. M Yu, A Rhuma, SM Naqvi, L Wang, J Chambers (2012) A posture recognition-based fall detection system for monitoring an elderly person in a smart home environment. In IEEE Transactions on Information Technology in Biomedicine 16(6): 1274-1286.

15. L Liu, M Popescu, KC Ho, M Skubic, M Rantz (2012) Doppler radar sensor positioning in a fall detection system. In 2012 Annual International
Conference of the IEEE Engineering in Medicine and Biology Society, San Diego, CA, pp. 256-259.

16. AK Bourke, Sandra P, Friedrch K, Victor C, Carlos T, et al. (2012) Embedded fall and activity monitoring for a wearable ambient assisted living solution for older adults. In 2012 Annual International Conference of the IEEE Engineering in Medicine and Biology Society, San Diego, CA, pp. 248-251.

17. K Niazmand, C Jehle, LT Dangelo, TC Lueth (2010) A new washable lowcost garment for everyday fall detection. In 2010 Annual International Conference of the IEEE Engineering in Medicine and Biology, Buenos Aires pp. 6377-6380.

18. SY Sim, HS Jeon, GS Chung, SK Kim, SJ Kwon, et al. (2011) Fall detection algorithm for the elderly using acceleration sensors on the shoes. In Annual International Conference of the IEEE Engineering in Medicine and Biology Society, Boston, MA, pp. 4935-4938.

19. R Narasimhan (2012) Skin-contact sensor for automatic fall detection. In Annual International Conference of the IEEE Engineering in Medicine and Biology Society, San Diego, CA, pp. 4038-4041.

20. C Park, J Suh, E Cha, H Bae (2011) Pedestrian navigation system with fall detection and energy expenditure calculation. In IEEE International Instrumentation and Measurement Technology Conference, Binjiang, $\mathrm{p}$. $1-4$.

21. Su YS, Twu SH (2020) A Real Time Fall Detection System Using TriAxial Accelerometer and Clinometer Based on Smart Phones. In: Lin KP, Magjarevic R, de Carvalho P (Eds.)., Future Trends in Biomedical and Health Informatics and Cybersecurity in Medical Devices. ICBHI 2019, IFMBE Proceedings, Springer 74.

22. M Tolkiehn, L Atallah, B Lo, G Yang (2011) Direction sensitive fall detection using a triaxial accelerometer and a barometric pressure sensor. In 2011 Annual International Conference of the IEEE Engineering in Medicine and Biology Society, Boston, MA, pp. 369-372.

23. Y He, Y Li, S Bao (2012) Fall detection by built-in tri-accelerometer of smartphone. In Proceedings of 2012 IEEE-EMBS International Conference on Biomedical and Health Informatics, Hong Kong, pp. 184187.

24. J Lee, H Tseng (2019) Development of an enhanced threshold-based fall detection system using smartphones with built-in accelerometers. In IEEE Sensors Journal 19(18): 8293-8302.

25. Y Bai, S Wu, C Tsai (2012) Design and implementation of a fall monitor system by using a 3-axis accelerometer in a smart phone. In IEEE 16th International Symposium on Consumer Electronics, Harrisburg, PA, p. $1-6$.

26. NM Kosse, K Brands, JM Bauer, T Hortobagyi, CJ Lamoth (2013) Sensor technologies aiming at fall prevention in institutionalised old adults: a synthesis of current knowledge. Int J Med Inform 82(9): 743-752.

27. RG Cumming, C Sherrington, SR Lord, JM Simpson, C Vogler, et al. (2008) Cluster randomised trial of atargeted multifactorial intervention to prevent falls among older people in hospital. In BMJ 336: 758-760.

28. (2020) Bank my cell website. New York,USA.

29. H Hawley Hague, E Boulton, A Hall, K Pfeiffer, C Todd (2014) Older adults' perceptions of technologies aimed at falls prevention, detection or monitoring: A systematic review. In Int J Med Inform 83(6): 416-426.

30. Kozina S, Gjoreski H, Gams M, Lustrek M (2013) Efficient activity recognition and fall detection using accelerometers. In Evaluating AAL Systems Through Competitive Benchmarking. EvAAL 2013, Communications in Computer and Information Science 386: 13-23.

31. R Igual, C Medrano, I Plaza (2013) Challenges, issues and trends in fall detection systems. In BioMed Eng OnLine 12(66): 1-66.

32. Radmanesh Elahe, Delrobaei Mehdi, Habachi Oussama, Chamani Somayyeh, Pousset Yannis, et al. (2020) A Wearable IoT-Based Fall 
Detection System Using Triaxial Accelerometer and Barometric Pressure Sensor 158-170.

33. Kumar VS, Acharya KG, Sandeep B, Jayavignesh T, Chaturvedi A (2019) Wearable Sensor-Based Human Fall Detection Wireless System. In: Zungeru A, Subashini S, Vetrivelan P (Eds.)., Wireless Communication Networks and Internet of Things. Lecture Notes in Electrical Engineering Springer, Singapore, pp. 493.

34. Islam M, Neom N, Imtiaz M, Nooruddin S, Islam M, et al. (2019) A review on fall detection systems using data from smartphone sensors. In Ingénierie des systèmes d information 24: 569-576.

35. Dharmitha Ajerla, Sazia Mahfuz, Farhana Zulkernine (2019) A RealTime Patient Monitoring Framework for Fall Detection. Wireless Communications and Mobile Computing 2019.

36. J Klenk, C Becker, F Lieken, S Nicolai, W Maetzler, et al. (2011) Comparison of acceleration signals of simulated and real-world backward falls. In Med Eng Phys 33(3): 368-373.

\section{ISSN: 2574-1241}

DOI: 10.26717/BJSTR.2021.34.005588

Chiara Bicchielli. Biomed J Sci \& Tech Res

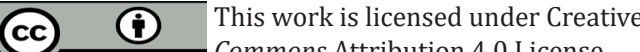

Submission Link: https://biomedres.us/submit-manuscript.php
37. F Ferri, DM Pisanelli, FL Ricci (1996) An object-oriented model for a multimedia patient folder. In ACM SIGBIO Newsletter 16: 2-18.

38. F Ferri (1995) The medical folder as an active tool in defining the clinical decision-making process. In Medical Informatics 20(2): 97-112.

39. A DUlizia, F Ferri, P Grifoni, T Guzzo (2010) Smart homes to support elderly people: Innovative technologies and social impacts. In Pervasive and Smart Technologies for Healthcare: Ubiquitous Methodologies and Tools, IGI Global p. 25-38.

40. SR Lord, JA Ward, P Williams, KJ Anstey (1993) An epidemiological study of falls in older community-dwelling women: The Randwick falls and fractures study. In Australian Journal of Public Health 17(3): 240-245.

\begin{tabular}{ll} 
BIOMEDICAL & Assets of Publishing with us \\
RESEARCHES & - Global archiving of articles \\
\hline & - Immediate, unrestricted online access \\
\hline
\end{tabular}

